\title{
Flow Mediated Vasodilation and Some Biomarkers of Endothelial Activation in Pre-Hypertensive
} Objects

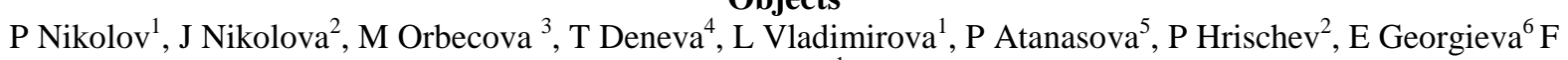
Nikolov ${ }^{1}$

\begin{abstract}
Pre-hypertension is a precursor of hypertension. Endothelial dysfunction is the key element for early prediction of cardiovascular events. We investigated whether flow mediated dilation, a non-invasive method for assessment of endothelial function, is decreased and if there is a parallel with some biomarkers of endothelial dysfunction. 103 patients with pre-hypertension at the age 43,5 \pm 6 years, were enrolled. Weight, body surface area, waist, total cholesterol, HDL-cholesterol, LDL-cholesterol, triglycerides, plasma glucose were followed up for each patient, indicating statistically higher values in the pre-hypertensive subjects. Flow mediated dilation was reduced when compared to our control data from healthy volunteers. It was in parallel with ADMA and sVCAM -1. There were no significant differences in sICAM-1. Pre-hypertension objects demonstrated reduced flow mediated dilation and significantly changed ADMA and sVCAM-1. Intima-media thickness didn't show any significant differences between pre-hypertensive and healthy objects. In conclusion, there is a correlation between clinical chemical biomarkers, flow mediated dilation, endothelial dysfunction and pre-hypertension which confirms their role as a predictor of pre-hypertention and cardiovascular disorders and as a challenge for primary prevention.
\end{abstract}

Keywords: Endothelial dysfunction, flow mediated dilation, pre-hypertension, endothelial dysfunction, flow mediated dilation, intima-media thickness, ADMA, sMCAM-1., sICAM-1., sVCAM -1.

From: ${ }^{1}$ First department of internal diseases - section of Cardiology, Medical University - Plovdiv, Bulgaria ${ }^{2}$ Department of Physiology, Medical University - Plovdiv, Bulgaria ${ }^{3}$ Second Department of Internal diseases Endocrinology, Medical University - Plovdiv, Bulgaria ${ }^{4}$ Central Clinical laboratory, Medical University Plovdiv, Bulgaria, ${ }^{5}$ Department of Anatomy, Histology and Embryology, Medical University - Plovdiv, Bulgaria ${ }^{6}$ Department of Developmental Biology - section of Embryology and Histology, Faculty of Biology, University of Plovdiv, Bulgaria, Elenka Stoilova Georgieva - Department of Developmental Biology - section of Embryology and Histology, Faculty of Biology, University of Plovdiv, 24 Tzar Assen Street, 4000, Plovdiv, Bulgaria, E-mail: e_tomova@abv.bg

Correspondence: ${ }^{5}$ Department of Anatomy, Histology and Embryology, Medical University - Plovdiv, Bulgaria ${ }^{6}$ Department of Developmental Biology - section of Embryology and Histology, Faculty of Biology, University of Plovdiv, Bulgaria, Elenka Stoilova Georgieva - Department of Developmental Biology - section of Embryology and Histology, Faculty of Biology, University of Plovdiv, 24 Tzar Assen Street, 4000, Plovdiv, Bulgaria, E-mail: e_tomova@abv.bg 


\section{INTRODUCTION}

Pre-hypertension (PH) includes arterial blood pressure (BP) values in its high referent limits and is concerned with eventual deviations in vascular homeostasis, endothelial dysfunction (ED) and early organs alterations. The term pre-hypertension is introduced in 2003 year by JNC-7 (Joint National Committee) - guideline for prevention, evaluation and treatment of hypertension in USA. The classified values for the systolic and/or diastolic blood pressure are 120-139and/or $80-89 \mathrm{mmHg},(1,2,3,4,5)$. The argumentation for introducing $\mathrm{PH}$ is in concern with the evidence based medicine. It is established that the increase of BP with $20 \mathrm{mmHg} / 10 \mathrm{mmHg}$ for systole and/or diastole respectively doubles the cardiovascular risk (6, 7), compared to normotensive objects. According to the Framingham heart study (8), people with $\mathrm{PH}$ have doubled probability to develop hypertension and following cardiovascular incidents in comparison to the normotensive. It is controversial nowadays, if $\mathrm{PH}$ values are normal or pathological one. According to the European Society of Hypertension (ESH) guidelines 2013, medical treatment for PH is not recommended.

Responding to or secreting both agonistic and antagonistic physiologically active substances, endothelium is the structure that modulates the vascular smooth muscle cells, producing relaxation or contraction, and therefore vasodilatation or vasoconstriction. Altered endothelial cells capacity within ED stimulates endothelial and blood cells interaction and the formation of adhesion cell molecules. Data, recently received shows that the increased cell adhesion molecules levels in healthy men and women are concerned with higher risk of acute cardiovascular incidents. Evidence based medicine shows that the methods for ED evaluation are considered useful in clinical practice $(9,10,11,12)$. Flow mediated dilation (FMD) detects changes in the conduit arterial diameter in relatively superficial arteries, such as the brachial, radial or femoral. Well standardized, flow mediated dilation (FMD) of the forearm brachial artery is an ultrasound noninvasive method for assessing ED with good correlation to 
cardiovascular complications. Ligating immunoglobulins, as serum intercellular cell adhesion molecule -1(sICAM-1) and serum vascular cell adhesion molecule (sVCAM-1), are some of the endothelial cell surface secreted physiologically active substances, circulating within the bloodstream (13). The measurement of the dissolved forms of the adhesion molecules in plasma or serum makes it possible their usage as biomarkers of early ED. Asymmetric methyl-arginine (ADMA) - structural analogue of aminoacid L-arginin, acts as endogenious inhibitor of endogenious nitric oxide synthetase (eNOS). Thus, the ADMA concentration is strongly concerned with the metabolism of L-arginin enzymes activity. ADMA, accumulating in vessel wall, is a reason for NO decreased availability and impaired endothelium dependent vasodilation. Being physiologic regulator of NO synthesis, according to many investigators, ADMA is supposed to be a crucial factor for testing ED in hypercholesterolemia, hypertension, diabetes mellitus, smoking, heart failure, erectile dysfunction $(14,15,16,17)$. Some epidemiologic and prospective investigations within last year's prove ADMA cardiovascular morbidity and mortality relationship $(18,19,20,21)$. Due to this, ADMA is discussed as a reliable marker for ED.

The goal of this study was ED to be assessed by FMD and ADMA, sICAM and sVCAM levels to be followed up in pre hypertensive objects and ED role to be evaluated as a predictor of $\mathrm{PH}$, compared to intima-media thickness data.

\section{MATERIALS AND METHODS}

148 men and women (103 with PH and 45 controls) from South Bulgaria at age of 18-64 years were followed up after dropping out of 33 objects with masked hypertension from a population of 181 persons. The protocol was approved by the Ethics Committee of the Medical University, Plovdiv. All study participants signed an informed consent. 
2.1. BP measuring was carried out with validated devices (Microlife, Switzerland) keeping the proper rules.

2.2. Weight, waist and body surface area and are measured too.

2.3. FMD was detected as the diameter of the forearm brachial artery of the nondominated hand was measured by high-resolution external vascular ultrasound device (Phillips 11XE) in response to an increased blood flow (causing shear-stress) during reactive hyperaemia (induced by cuff inflation and then deflation). This leads to endotheliumdependent dilatation. The artery was scanned and the brachial artery diameter was measured during three conditions; baseline (after at least 10 min supine rest), during reactive hyperaemia, induced by inflation to $250 \mathrm{mmHg}$ and then deflation of a sphygmomanometer cuff around the forearm. The time required to obtain a high quality baseline scan varies between 1 and $10 \mathrm{~min}$. The cuff inflation period of $5 \mathrm{~min}$ was initially decided to produce adequate hyperaemia to allow flow-mediated dilatation, but not to compromise patient comfort. Shorter inflation periods do not seem to produce significant FMD. The usual scanning period used in our laboratory was $30 \mathrm{~s}$ before and $90 \mathrm{~s}$ after the cuff deflation. The measurements were made up with the help of automatic program Q-lab.

2.4. Intima-media thickness (IMT) of the common carotid artery (CCA) is an accessible method performed by high-resolution external vascular ultrasound device (Phillips 11XE). The image is transferred to a computer and by automatic analyzing system Q-lab measurements are done and the variability of the results are reduced.

2.5. ADMA and cell adhesion molecules (sVCAM-1 and sICAM-1) were determined by enzyme connected immune sorbent analysis (ELISA) with multi parameter clinic chemical ELISA Reader - SIRIO, SEAC, Italy.

2.6. Clinic chemical parameters as: blood sugar level (GLU) (RA 1000 Technicon, USA) and lipid profile (Optima KONE) - total cholesterol (TC), cholesterol with high 
density (HDL-C), cholesterol with low density (LDL-C) and triglycerides (TRY) were followed up, too.

Data were statistically worked up by SPSS package, version 17.0. The results were expressed as mean $\pm \mathrm{SD}$ at $\mathrm{p}<0.05$

\section{RESULTS}

The measured BP in PH was: SBP: 124,72 $\pm 8,67$ and DBP: $80 \pm 11 \mathrm{mmHg}$. Weight, body surface area, waist, total cholesterol, HDL-cholesterol, LDL-cholesterol, triglycerides, plasma glucose were statistically higher in the pre-hypertensive subjects. The characteristics of the followed up anthropometric and clinic chemical parameters within the groups is represented in table 1.

Based on the accepted in 2009 year diagnostic criteria for Metabolic syndrome (MS), the presence of at least 3 of the following 5 indices are necessary to be included: visceral obesity (waist $\geq 94 \mathrm{sm}$ in men and $\geq 80 \mathrm{sm}$ in women), increased triglycerides, decreased HDL-cholesterol or treatment of dyslipidemia, dysglicaemia /diabetes mellitus and ABP values above 130/85 mmHg or treated hypertension. Our data showed that Pre-hypertensive objects with MS were $61, \%(\mathrm{p}=0,004)$.

When compared, FMD in Pre-hypertensive and Normotensive objects, it is seen that in $\mathrm{PH}$ objects the percentage of FMD is significantly decreased - FMD\% in PH objects is $10,75 \pm 9,55$ vs $16,07 \pm 8,75(\mathrm{p}<0,05)$ in the Normotensive (table 2$)$.

Intima-media thickness (IMT) didn't show any significant differences between prehypertensive and healthy objects. IMT of the right and left common carotid artery (CCA) didn't show any significant differences between pre-hypertensive and healthy objects (RCCA-0,55 $\pm 0,14$ and LCCA-0,56 $\pm 0,12$ in PH). Flow mediated dilation was in parallel with ADMA and sVCAM -1. Pre-hypertension objects demonstrated reduced flow mediated 
dilation and significantly changed ADMA and sVCAM-1. According to the followed up cell adhesion molecules in PH objects the data showed that sVCAM were significantly higher in comparison with normotensive objects $(1041,90 \pm 238,9$ vs $686,5 \pm 153,44, \mathrm{p}<0,05)$ while ICAM was non significantly changed $(553,05 \pm 126,82$ vs $471,0 \pm 32,52$ in Normotensive respectively). ADMA in $\mathrm{PH}$ objects was also significantly higher $(1,03 \pm 0,39$ vs $0,71 \pm 0,18$, $\mathrm{p}<0.05$ in Normotensive respectively). Dynamically changed endothelial activation testing markers in PH and normotensive objects are demonstrated in table 2.

\section{Discussion}

The percentage of pre-hypertensive objects is higher in Europe (40-50\%) than in USA. The highest is the share of pre-hypertensive objects among 30-39 years as their percentage decreases with age due to its progression in hypertension. The same is thetendency in our study. Overweight is the major risk factor for the PH. People with overweight and especially these with visceral type of obesity are with higher risk of $\mathrm{PH}$ and hypertension. It is established that even $1 \mathrm{~kg}$ body mass loss leads to reduction in systolic and diastolic BP with $1 \mathrm{mmHg}$. We accept that having in mind the anthropometric parameters, the waist is the major predictor of $\mathrm{PH}$ and hypertension. The visceral obesity plays an independent role in raising $\mathrm{ABP}$ in all the patients with dyslipidaemia. Dyslipidaemia leads to $\mathrm{ED}$, loss of vessel wall vasomotor reactivity and increased arterial wall rigidity. In our study, there are significant differences in TC, LDL-C and TRY of the pre-hypertensive in comparison to the normotensive objects. Cardiovascular risk factors as hypercholesterolemia, hypertension, smoking, diabetes, sedentary, alter the endothelial cells capacity and lead to ED. ED is the first step within the clinical development of many cardiovascular events, and in this aspect, of $\mathrm{PH}$ too. The vasodilator/vasoconstrictor balance is impaired, the production and/or NO availability are depressed and the secretion of cell adhesion molecules increased. Our data 
conferms that of other authors (22) that serum adhesion cell molecules and ADMA are in direct relationship and are of predispose manner to ED. In our study, ADMA is significantly expressed in the followed up pre-hypertensive objects in comparison to the normotensive one. The increased level of ADMA is concerned with decreased production of NO, giving the start of ED. Vascular endothelium is the organ chiefly responsible for the catabolism of plasma ADMA. Thus, ADMA could be a reliable mediator of ED $(22,20,23)$. It could be regarded to as marker of $\mathrm{PH}$ and vascular disease as a whole.

The increased levels of TC and especially these of LDL cholesterol suppressing NO, lead to increased expression of cell adhesion molecules. It is established that patients with hypercholesterolemia express significantly high levels of sVCAM-1, sICAM-1 and E-selectin in comparison to the control group. sVCAM-1 is an independent prognostic indicator for risk of coronary incidents after acute coronary syndrome $(24,8)$. In our study sVCAM-1 is significantly raised compared to normotensives. In this aspect we think that $\mathrm{PH}$ in constilation with dislipidemia and increased expression of cell adhesion molecules is a prognostic indicator for eventual coronary incidents.

Impaired FMD reflects endothelial function activation. In our study, the detected vessel wall changes in $\mathrm{PH}$, being the precursor of clinical hypertension, are still, functional but not structural. Within the last years it was established that FMD is one of the most sensitive parameters for measuring ED in PH. ED increases its value making it usefull for follow-up non-invasively the progression of the endothelial activation and the effects of different treatments. IMT of the CCA is a reliable method for the vascular changes assessment in the peripheral circulation, being thus one of the predictors of the big cardiovascular incidents. IMT above $1,2 \mathrm{~mm}$ is a certain sign of atherosclerotic changed vessel wall. 
In conclusion: ED indicates a higher risk for PH objects. The results of the present study are a possibility for correlation between clinic chemical biomarkers, FMD and PH and their role as predictors for new therapeutic approaches. Early intervention and life style modification may improve endothelial function and increase FMD and change some ED biomarkers levels. 


\section{REFERENCES}

1. Chobanian AV , Bakris GL, Black HR, Cushman WC, Green LA, Izzo JL et al. The seventh report of the Joint national Committee on Prevention, Detection, Evaluation and Treatment of High Blood Pressure. Hypertension 2003; 42: 1206-1252.

2. Kaplan N. Kaplan's Clinical Hypertension - ninth edition, Philadelphia, Lippincott Williams \& Wilkins. 2006; 11-12.

3. Mancia G, Laurent St, Agabiti-Rosei E, Ambrosioniet E, Burnier M, Caulfield MJ et al. Reappraisal of European guidelines of hypertension management. J Hypertens 2009; 27: 2121-2158.

4. Fagard R. Reappraisal of the EUROPEAN guidelines on hypertension management. The European Society of Hypertension. Task Force document; a short review. Pol Arch Med Wien 2010; 120: 31 .

5. Kearney PM, Whelton M, Reynolds K, Muntner P, Whelton PK, He J. Global burden of hypertension: analysis of worldwide data. Lancet 2005; 365: 217-23.

6. Wolf-Maier K, Cooper RS, Banegas JR, Giampaoli S, Hense HW, Joffres M et al. Hypertension prevalence and blood pressure levels in 6 European countries, Canada and the United States. JAMA 2003; 289: 2363-9.

7. Ramachandran S, Vasan MD, Martin G, Larson ScD, Eric P, Leip MS et al. Impact of high-normal blood pressure on the risk of cardiovascular disease. N Engl J Med 2001; 345: 1291-1297.

8. Vasan RS, Larson MG, Leip EP, Kannel WB, Levy WD. Assesment of frequency to progression in non-hypertensive participants in the Framingham Heart Study: a cohort study. Lancet 2001; 358: 1682-1686.

9. Coretti C, Anderson T, Benjamin EJ, Celermajer D, Charbonneau F, Creager MA et al. Guidelines for the ultrasound assessment of endothelial dependent flow-mediated 
vasodilatation of the brachial artery: a report of the International Brachial Artery Reactivity Task Force. J Am Coll Cardiol 2002; 39: 257-265.

10. Quyyumi AA, Prognostic value of endothelial function. Am. J Cardiology 2003; 91: 1924.

11. Poredos P. Endothelial dysfunction in the pathogenesis of atherosclerosis. Int Andiol 2002; 21:109-16.

12. Verma S, Buchnan MR, Anderson TJ. Endothelial function testing as a biomarker of vascular disease. Circulation 2003; 108: 2054-9.

13. Pradhan AD, Rifai N, Ridker PM. Soluble intercellular adhesion molecule -1, soluble vascular adhesion molecule-1, and the development of symptomatic peripherial arterial disease in men. Circulation. 2002; 106: 820-826.

14. Schnabel R, Blankenberg S, Lubos E, Lackner KJ et al. Asymmetric dimethylarginine and risk of cardiovascular events and death in patients with coronary artery disease: results from the AtheroGene Study. Circ Res 2005; 97: 53-59.

15. Schulze F, Wessmman R, Schwedhelm E, Sydow K, Albsmeier J, Cooke JP et al. Determination of asymmetric dimethylarginine (ADMA) using ELISA. Clin Chem.Lab Med 2004; 42: 1377-1383.

16. Schulze F, Maas R, Freese R, Schwedhelm E, Silberhorn E, Boger RH. Determination of reference value for N, N- dimethyl-L-arginine in 500 subjects. Eur J Clin Invest 2005; 35: $622-626$.

17. Stuhlinger MC, Oka RK, Graf EE, Schmölzer I, Upson BM, Kapoor O, Endothelial dysfunction induced by hyperhomocyst(e)inemia: role of asymmetric dimethylarginine. Circulation 2003; 108: 933-938.

18. Böger RH. The emerging role of asymmetric dimethylarginine as a novel cardiovascular risk factor. Cardiovas Res 2003a; 59: 824-33. 
19. Böger RH, Vallance P, Cooke J. Asymmetric dimethylarginine (ADMA): a key regular of nitric oxide synthase. Atherosclerosis Suppl 2003; 4: 1-3.

20. Böger RH, Lenzen H, Hanefeld C. Asymmetric dimethylarginine and the risk of coronary heart disease: relationship with tradicional risk factor as assassed in the multicenter CARDIAC study [Abstract]. J Am Coll Cardiol (suppl) 2004: 515A.

21. Lu TM, Ding YA, Lin SJ, Lee WS, Tai HC. Plasma levels of asymmetrical dimethylarginine and adverse cardiovascular events after percutaneous coronary intervention. Eur. Heart. J. 2003; 24: 1912-19.

22. Mc Carty MF. Vascular endothelium is the organ chieftly responsible for the catabolism of plasma asymmetric dimethylarginine - an explanation for the elevation of plasma ADMA in disordes by endothelial dysfunction. Med Hypotheses 2004; 63: 699-708.

23. Böger R H. Asymmetric dimethylarginine (ADMA) modulate endothelial functiontherapeutic implication. Vasc Med 2003b; 8: 149-151.

24. Vallance P, Leone A, Calver A, Collier J, Moncada S. Endogenous dimethylarginine as an inhibitor of nitric oxide synthesis, J Cardiovasc Pharmacol 1992; 20: 60-62. 
Table 1: Anthropometric and clinic chemical parameters followed up in $\mathrm{PH}$ and Normotensive groups.

\begin{tabular}{|c|c|c|c|}
\hline INDECES & $\begin{array}{l}\text { PH } \\
(\mathbf{1 0 3})\end{array}$ & $\begin{array}{l}\text { NORMOTENSIVE } \\
(45)\end{array}$ & $\mathbf{P}$ \\
\hline Age & $43,5 \pm 6$ & $41,2 \pm 7$ & NS \\
\hline Gender & $58 \overbrace{}^{\top} / 45$ 우 & $24 \sigma^{\top} / 21$ 우 & NS \\
\hline Weight (kg) & $104,88 \pm 15,24$ & $75,57 \pm 22,46$ & $<0,01$ \\
\hline Body surface area (m2) & $2,08 \pm 0,12$ & $1,85 \pm 0,27$ & $<0,01$ \\
\hline Waist (sm) & $106,12 \pm 10,25$ & $82,43 \pm 15,80$ & $<0,01$ \\
\hline Total cholesterol (TC) (mmol.L-1) & $5,49 \pm 1,6$ & $4,03 \pm 1,48$ & $<0,05$ \\
\hline Triglycerides (mmol.L-1) & $2,08 \pm 1,38$ & $1,13 \pm 1,16$ & $<0,05$ \\
\hline HDL -cholesterol (mmol.L-1) & $1,07 \pm 0,19$ & $1,31 \pm 0,16$ & NS \\
\hline LDL-cholesterol (mmol.L-1) & $3,46 \pm 1,05$ & $2,25 \pm 1,05$ & $<0,05$ \\
\hline Plasma Glucose (mmol.L-1) & $5,12 \pm 0,6$ & $4,74 \pm 0,56$ & NS \\
\hline
\end{tabular}

Table 2: Dynamically changed endothelial activation testing markers in $\mathrm{PH}$ and normotensive objects.

\begin{tabular}{llll}
\hline MARKERS & PREHYPERTENSIVE & NORMOTENSIVE & P \\
\hline FMD\% & $10,75 \pm 9,55$ & $16,07 \pm 8,75$ & $<0,05$ \\
ADMA ( $\boldsymbol{\mu g . L - 1 )}$ & $1,03 \pm 0,39$ & $0,71 \pm 0,18$ & $<0,05$ \\
SVCAM (ng.ml-1) & $1041,90 \pm 238,9$ & $686,5 \pm 153,44$ & $<0,05$ \\
\hline
\end{tabular}

